\title{
Apologie Pour les Faiseurs de Modèles
}

À propos de Guillaume Tusseau, Modelli di giustizia costituzionale. Saggio di critica metodologica-Contre les « modèles » de justice constitutionnelle. Essai de critique méthodologique, Bononia University Press, Coll. « Ricerche di Diritto Comparato », Bologna, 2009, 106 p. -87 p. pour la partie française.

\section{Jean-Baptiste Pointel}

Published online: 12 February 2011

(c) The Author(s) 2011. This article is published with open access at Springerlink.com

Résumé Dans son ouvrage Contre les «modèles » de justice constitutionnelle. Essai de critique méthodologique, Guillaume Tusseau décide de déconstruire et de combattre la traditionnelle dichotomie entre le soi-disant «modèle américain » et celui «européen» ou «kelsénien». En effet, cette distinction est inutile voire contre-productive. Cet écrit est, pour l'auteur, l'occasion d'énoncer un projet comparatif, s'affranchissant des cadres antérieurs. Si sa démonstration est convaincante et fortement argumentée, une autre position théorique peut toutefois être embrassée. Sans être opposée à celle développée dans l'ouvrage, elle peut se présenter de manière alternative, voire complémentaire. Celle-ci ouvre alors deux pistes de réflexion : la définition du concept de modèle et une remise en cause radicale des critères employés actuellement par la doctrine. Soutenus par une démarche problématologique, nous appelons à l'avènement d'un nouveau paradigme plus pragmatique et questionnant, que l'on pourrait qualifier de « post-moderne ».

Mots-clés Justice constitutionnelle $\cdot$ Constitution $\cdot$ Modèle $\cdot$ Guillaume Tusseau . Problématologie

1. Compte tenu du foisonnement des publications sur les modèles de justice constitutionnelle, apparaît une certaine fébrilité dès lors qu'il s'agit d'aborder le livre de Guillaume Tusseau. L'intitulé paraît impliquer une attaque frontale et-semble-t-il—radicale au concept de modèle, cet outil incontournable du contentieux constitutionnel comparé contemporain. Or, nonobstant le titre de

Il est fait référence au fameux article de Jean Rivero : « Apologie pour les faiseurs de systèmes », D. [24], Chronique, p. 99.

\section{J.-B. Pointel $(\bowtie)$}

Faculté de Droit, Centre d'Études des Systèmes Juridiques, 3 Avenue Pasteur,

76186 Rouen Cedex 1, France

e-mail: jean-baptiste.pointel@univ-rouen.fr 
l'ouvrage, et comme précisé dès la préface par Lucio Pegaro, Guillaume Tusseau « ne nie pas, à [son] avis, contrairement à ce qu'il semble évoquer, l'utilité de la construction de modèles. » (p. 9) Au contraire, son argumentation vise à contester la dichotomie « traditionnelle » qui prévaut au sein de la recherche en contentieux constitutionnel : l'opposition dogmatique du modèle américain au modèle européen ou kelsénien.

2. La démonstration de l'auteur est extrêmement convaincante : la distinction binaire et apparemment immanente entre ces deux modèles de justice constitutionnelle est inutile, du moins contre-productive. Certes, les critères d'opposition classique ${ }^{2}$ offrent une construction correcte sur le plan logique (Introduction, not. pp. 20-23). Toutefois, ils ne permettent pas d'embrasser l'intégralité du droit positif : cela fait abstraction des pays ayant un mécanisme «mixte » panachant les critères voire parfois les cumulant. Ils ne participent pas non plus à une explication rigoureuse de la réalité : il n'existe pas de liens autres que conjoncturels entre ces différents critères, dont les modèles auraient pu porter la signification d'ensemble. La persistance de ces modèles dans le débat international ne peut donc se justifier au regard de leur qualité scientifique.

3. Il convient dès lors de la rechercher dans leur origine. Sur cet autre aspect, la réponse apportée peut apparaître surprenante : les modèles n'ont été édifiés que récemment (milieu des années 1980) et procèdent de reconstruction des œuvres de Marshall, mais surtout de $\operatorname{Kelsen}^{3}$ (p. 61 et s., not. p. 66.) par le biais de la thèse d'Eisenmann, rééditée en 1986. ${ }^{4}$ Guillaume Tusseau identifie ses deux principaux artisans, l'un politique, Robert Badinter, l'autre juriste de renom, Louis Favoreu. Leur objectif aurait été de défendre le Conseil constitutionnel français comme une véritable cour constitutionnelle, n'ayant plus à rougir devant le juge américain. Ainsi, la juridiction française, auparavant décriée, se serait transformée en une variante du genre de la justice constitutionnelle, au point même de devenir l'archétype du «modèle européen ». Ceci permit tant une revendication disciplinaire-la suprématie $\mathrm{du}$ droit constitutionnel dans le champ juridique-qu'une revendication politique-présenter la France comme un idéal à imiter. Cette démarche politico-idéologique serait l'unique explication du succès des modèles. Elle ne correspond cependant pas aux canons de la science du droit.

4. Il est par conséquent indispensable de « repenser l'étude comparée du contentieux constitutionnel » (Titre du chapitre III, p. 71). Pour tirer les conclusions de ce constat, deux voies s'opposent: rétablir des modèles

\footnotetext{
1 Toutefois, il semble qu'implicitement une attaque soit portée au concept même de modèle, dans une certaine manière de l'appréhender, v. infra.

2 Ainsi, sont opposés : contentieux concentré ou déconcentré ; abstrait ou concret ; a priori ou a posteriori, par voie d'action ou d'exception ; avec un effet erga omnes ou inter partes.

3 En mettant en avant son article « Judicial Review of Legislaton. A Comparative Study of the Austrian and the American Constitution », The Journals of Politics, Vol. 4 [13], pp. 183-200.

4 Eisenmann, C., La justice constitutionnelle et la Haute Cour constitutionnelle d'Autriche, av.-prop. Vedel, G., préf. Kelsen, H., Annexe Favoreu, L., Paris, Economica, PUAM, coll. « Droit public positif », 1928, rééd. [9], xvii+xiv+383 p.
} 
efficaces - soit en leur adjoignant un tertium genus, voire un quartum genus (pp. 72-73); soit en leur substituant des modèles fondés sur d'autres critères plus englobants (pp. 74-76)—; ou bien procéder à une nouvelle classification, « renouvel[ant] de manière radicale l'approche du contentieux constitutionnel comparé » (p. 77). La première solution apparaît soit comme une quête vaine qui consisterait à poser des rustines dans un bateau qui prend l'eau de toutes parts, soit comme une manière de repeindre l'ancien pour le parer d'habits neufs, mais sans réellement s'attaquer aux causes de l'échec du modèle et de se prêter aux mêmes critiques-voire davantage : en quoi l'unique critère sélectionné est-il plus pertinent qu'un autre? Bien sûr, la position privilégiée par Guillaume Tusseau est la seconde, celle radicale alliant théorie du droit et droit comparé (p. 80 et s.). Il annonce alors un « nouveau programme » (p. 80), celui d'une théorie analytique conforme à son projet benthamien, c'est-à-dire : « élaborer une grille générale des dispositifs existants de par le monde » (p. 86). Les critères seraient définis in abstracto de sorte à être logiquement corrects et permettre « une utilisation strictement descriptive et scientifique. » (p. 83) Ils seraient, ainsi, non contingents à une époque ni à un cadre géographique et culturel donné. Aussi, il est possible d'éviter l'ethnocentrisme et les idéologies « clandestines » tout autant que de prévoir des évolutions institutionnelles. « Il s'agit donc de bâtir un métalangage neutre, indépendant des langages-objets analysés, et susceptible de subsumer les catégories employées par chacun d'eux [...] sous des concepts théoriques plus généraux » (p. 84).

5. Cependant, après la lecture de l'ouvrage, une gêne subsiste. L'objectif de Guillaume Tusseau est de prendre position dans un débat international et de justifier à la fois son projet de renouveau du contentieux constitutionnel comparé et sa démarche manifestement analytique, inscrite dans la théorie réaliste de l'interprétation. Il s'attaque alors à une fiction (les « modèles » de justice constitutionnelle) et la déconstruit comme un château de cartes. Si sa démonstration est vivement convaincante et fortement argumentée, une autre position théorique peut toutefois être embrassée. Sans être opposée à celle développée dans l'ouvrage, elle peut se présenter de manière alternative, voire complémentaire. Celle-ci ouvre alors deux pistes de réflexion : la définition du concept de modèle et une remise en cause radicale des critères employés actuellement par la doctrine.

6. Tout d'abord, l'objet ciblé au cœur de l'étude demeure flou : si les « modèles de justice constitutionnelle » sont définis en ce que ses deux manifestations recoupent, il n'est pas procédé à une analyse du concept même de « modèle »—tel n'était toutefois pas l'objet de l'étude et ne présentait pas d'intérêt pour la démarche suivie. ${ }^{5}$ L'utilité du modèle est brièvement présentée, immédiatement rejetée, du moins pour ce qui est de la science du droit. Le modèle serait-il donc constitutif d'un outil non juridique? ${ }^{6}$

\footnotetext{
${ }^{5}$ Une vision d'ensemble est proposée dans les premières pages de l'ouvrage, mais visent davantage à cerner le sujet en introduction. (pp. 17-20).

${ }^{6}$ En effet, un modèle n'est pas du droit positif. S'il n'a pas d'intérêt en science du droit, quel serait son intérêt pour l'étude du droit?
} 
Guillaume Tusseau oppose au concept de modèle une critique classique et traditionnelle qu'affectionnent les partisans d'une épistémologie relativiste (p. 81) : la dénonciation réductrice au platonisme supposé des défenseurs des modèles (pp. 50-51)—certes, il faut le concéder, une telle croyance est implicite chez certains auteurs. Or, il n'est pas nécessaire, lorsqu'on pose une idée, qu'elle fasse écho à une ontologie forte, c'est-à-dire qu'un concept préexisterait dans la réalité indépendamment de tout travail conceptuel. ${ }^{7} \mathrm{Au}$ contraire, il est tout à fait possible de poser, comme convention de langage, un concept et ultérieurement de l'employer pour décrire le réel. Ainsi, le concept de licorne n'implique aucunement qu'il existe ontologiquement un tel animal. ${ }^{8}$ Il s'agit seulement d'une idée maniable qui répond à des questionnements, c'est-à-dire une métaphore. Bien évidemment, ces derniers siècles, la métaphore a subi de sévères oppositions, la disqualifiant d'office pour tout travail scientifique. ${ }^{9}$ Il faudrait alors aussi dénoncer la pyramide de Kelsen et la bouche de la loi de Montesquieu. Le renouveau de la rhétorique au $\mathrm{XX}^{\mathrm{e}}$ siècle a permis à cette technique de devenir un mode de raisonnement valable. La critique du platonisme est donc à rejeter.

7. Après avoir procédé à une déconstruction des deux modèles étudiés, avoir observé leur formation, les motivations sous-jacentes et les critères employés, Guillaume Tusseau met un terme à son analyse, en disqualifiant ce type de démarche. ${ }^{10}$ (p. 70) Le concept de modèle présente un intérêt pour la science du droit : par sa clarté d'explication et son économie d'exposition, ${ }^{11}$ il permet de mettre en lumière un problème et de soulever davantage des questions que des réponses. Il s'agit d'un concept à rapprocher des idéaux types de Max Weber. ${ }^{12}$ Ils n'ont pas vocation à se manifester dans le droit positif, mais à

\footnotetext{
7 V. par ex. Meyer, M., De la problématologie, Paris, PUF, Coll. « Quadrige » [15], p. 88; Id., Pour une histoire de l'ontologie, Paris,PUF, coll. «Quadrige» [16], 176 p, not. chap. I « La naissance de l'ontologie ».

${ }^{8}$ Heidegger, M, Logik als die Frage nach dem Wesen der Sprache, Francfort, Klostermann Vittorio $\mathrm{GmbH}$, coll. « Gesamtausgabe II » $\mathrm{n}^{\circ} 38$ [12], p. 142. V. aussi Quine, W. V. O., " On what there is », From a logical point of view, New York, Harper Torchbooks, 1953, 2e éd. [22], p. 13.

${ }^{9}$ Que ce soit les réalistes tel Platon (Gorgias, 465b-c) ou les empiristes tels Locke (Essai sur l'entendement humain, 1, III, Chap. X, trad. Coste, réimp. Paris, Vrin, 1983, p. 412) et Hobbes (Leviathan, Sirey, 1971, p. 44), la métaphore est perçue comme trompeuse et embrouillant la raison par son caractère esthétique. Elle est encore dénoncée par la philosophie analytique et l'empirisme logique en ce qu'elle perturbe le langage et va à l'encontre de l'objectif même de pureté de la réflexion.

${ }^{10}$ Pondérons : seuls les modèles dans leur manifestation en justice constitutionnelle sont rejetés. En effet, Guillaume Tusseau écrit que « la construction de « modèles » intégrant les éléments épars du droit positif dans un nombre limité de trames relativement cohérentes s'avère une voie prometteuse. » (p. 17).

11 Zoller, E., «Qu'est-ce que faire du droit constitutionnel comparé ? », Droits [33], n 32, p. 122; Boyer, P.-X., La réception des modèles étrangers dans le droit constitutionnel français. Grande Bretagne et Etats-Unis au prisme de la réflexion française, Thèse [4], Rouen, p. 24. Sur ce point, l'analyse de Guillaume Tusseau converge (p. 1 not.).

12 François Ost les définit très clairement : « Rappelons que, pour M. Weber, un type-idéal est un tableau de pensées qui réunit des relations et des événements déterminés de la vie historique en un "cosmos non contradictoires de relations pensées". Il s'agit d'une « utopie rationnelle » que l'on obtient en accentuant par la pensée des éléments déterminés de la réalité et en enchaînant une multitude de phénomènes. »: Ost, F. «Quelle jurisprudence, pour quelle société », in Id., Dire le droit, faire justice, Bruxelles, Bruylant, coll. «Penser le droit» [20], p. 4. V. aussi : Weber, M., Essais sur la théorie de la science,
} 
offrir une grille de lecture compréhensible dans un monde complexe. Il est superflu de vouloir opposer aux métaphores leur non-respect du réel. À l'inverse, la critique de son inadéquation explicative est pertinente. Comment un vecteur de questionnement peut-il sombrer dans l'apocritique ${ }^{13}$ ? En « s'endormant» ou en «mourant », c'est-à-dire en se lexicalisant. ${ }^{14}$ Une métaphore vive maintient son efficacité. Elle se réactualise sans cesse-un modèle doit s'adapter s'il ne veut mourir ${ }^{15}$ _, mais elle doit demeurer dans le champ de la métaphore. Le risque, et c'est là sa mort, est de la comprendre de manière littérale. C'est ainsi que s'est forgé le lit de la métaphysique. ${ }^{16} \mathrm{En}$ confondant le problématologique ${ }^{17}$ avec l'apocritique, nous procédons à un renversement entier de la métaphore. Toutefois, il ne faut pas chercher les

\section{Footnote 12 continued}

trad. Freund, J., Paris, Plon, Coll. « Recherches en sciences humaines » n 19 [32], p. 179-186 ; cf. aussi, pour l'explication du type idéal, Bourdieu, P., Chamboredon, J.-C. et Passeron, J.-C., le métier de sociologue, Paris, Mouton-Bordas [3], pp. 79 et s. V. aussi Badiou, A., Le concept de modele, Paris, Maspero [2], p. 14, pour qui le modèle est « un objet artificiel [...] il appartient au registre de l'invention pure ».

13 Le terme « apocritique » provient du vocabulaire spécifique forgé par Michel Meyer. Il le définit comme suit : «Une réponse apocritique, comme je l'appelle, clôt l'enquête, refoule le problématique et s'en détache plutôt qu'elle ne le fouille, l'explore et le met en lumière. Elle sert de base à une autre question, et ainsi de suite. Les questions disparaissent, les réponses s'accumulent. », Meyer, M., De la problématologie, op cit., p. 17. Il faut l'opposer au problématologique : v. infra note 18.

${ }^{14}$ Ce sujet est au cœur du travail d'Heidegger (op cit.) et entraînera une controverse très riche entre Paul Ricoeur et Jacques Derrida: Derrida, J., «La mythologie blanche. La métaphore dans le texte philosophique », in Id., Marges de la philosophie, Paris, Éd. de Minuit [7], pp. 247-324 ; Id., « Le retrait de la métaphore », Psychée. L'invention de l'autre, Paris, Galilée [8], pp. 63-93; Ricoeur, P., La métaphore vive, Paris, le Seuil, Coll. « L'ordre philosophique » [23], 414 p. Pour un exposé synthétique de ce «dialogue », v. Amalric, J.-L., Ricoeur, Derrida. L'enjeu de la métaphore, Paris, PUF, coll. «Philosophies » [1], $152 \mathrm{p}$.

15 Sacco, R., La comparaison juridique au service de la connaissance du droit, Paris, Economica, coll. «Études juridiques comparatives » [26], pp. 113-128. V. aussi Meny, Y. (Dir.), Les politiques du mimétisme institutionnel. La greffe et le rejet, Paris, L'Harmattan, coll. « Logiques politiques » [14], 285 p. ; Boyer, P.-X., La réception..., op cit., not. pp. 42-43 et p. 63.

16 Pour reprendre les termes de Michel Meyer, il y a alors un refoulement problématologique. Les identités sont perçues comme fortes. Le problématique envahit les réponses, tendant à une indifférenciation entre les deux niveaux. La confusion crée alors le fondement des problèmes métaphysiques. Pour de plus longs développements, v. Meyer, M., Pour une histoire de l'ontologie, op cit. De même, Derrida, «La mythologie blanche... », op cit., p. 254 : «Mythologie blanche-la métaphysique a effacé en ellemême la scène fabuleuse qui l'a produite et qui reste néanmoins active, remuante, inscrite à l'encre blanche, dessin invisible et recouvert dans le palimpseste. »; V. aussi : Nietzsche, F., Livre du philosophe, trad. Kremer-Marietti, A., Paris, Aubier-Flammarion [18], pp. 181-182 ; Amalric, J.-L., Ricoeur, Derrida. L'enjeu de la métaphore, op cit., p. 31 : «Pour Heidegger, la métaphore a partie liée avec la métaphysique car elle est une transposition d'un sens propre vers un sens figuré, qui a elle-même son origine dans l'opération de transport du visible à l'invisible caractéristique de la métaphysique occidentale. ».

17 La philosophie problématologique de Michel Meyer se construit en grand part autour de cette notion de «problématologique ». Il faut donc y apporter une grande attention : «Une réponse problématologique a ceci de particulier qu'elle fracture l'impensé de la pensée, marque des alternatives, crée un espace de relation et de sens, et que c'est cela la solution qu'elle apporte. » : Meyer, M., De la problématologie, op cit., p. 17. À opposer à la notion d' " apocritique », v. supra note 14. 
modalités aléthiques ${ }^{18}$ d'un modèle : il produit ses propres conditions de succès. Par conséquent, un modèle fondé sur une description erronée-celle de Montesquieu du régime anglais_-peut s'avérer très fructueux. Ce « modèle anglais » a été notamment utilisé par les constituants américains, pourtant fins connaisseurs de l'Angleterre du XVIII ${ }^{\mathrm{e}}$ siècle. $^{19}$

8. Une fois acquis le caractère métaphorique du modèle, il est possible de procéder à une définition de ce concept malléable et fécond. Il doit réunir trois caractéristiques : différenciation, standardisation, reproduction. ${ }^{20}$ Autrement dit, un modèle est à considérer comme problématisation : il est descriptif et contingent à une époque. Il doit alors s'actualiser sous peine de se confondre et de perdre son caractère distinctif, son identité ou son éthos. ${ }^{21}$ Cela lui offre une facilité d'exposition; le modèle est mimétique : il sert comme outil de reproduction $^{22}$ et n'existe qu'à partir du moment où il est employé de manière

\footnotetext{
18 Provenant de la théorie logique d'Aristote, une modalité dite « aléthique » définit un rapport de «vérité » avec une proposition, ou encore de «vérité objective ». Elle se place donc sur le plan du « nécessaire » ou de l'« impossible » de ce qui peut se produire. Une telle modalité décrit non seulement ce qui est mais aussi ce qui doit être. Ainsi, sont aléthiques les deux énoncés suivants :
}

Tous les hommes sont mortels (nécessité);

Un homme peut vivre 100 ans (possibilité).

Par contre, ne le sont pas les suivants:

Un homme a le droit de vivre jusqu'à l'âge de 80 ans (permission);

Il est interdit à un homme de vivre plus de 80 ans (interdiction);

Il devrait vivre au moins 80 ans (plausible);

Il y a 100 ans, un homme vivait en moyenne 50 ans (modalité temporelle);

Albert croit qu'un homme vit 90 ans (croyance personnelle); etc.

19 « [L]e droit étranger peut être influent même quand il est complètement mal-compris [...]. Il est raisonnable, bien qu'injuste, de se référer ici à Montesquieu et à la doctrine de la séparation des pouvoirs. Il est fortement contesté de savoir si Montesquieu a en réalité mal compris la constitution anglaise ou s'il était en train de construire une constitution idéale avec l'Angleterre comme sa source. Quoique cela puisse être, ses visions furent d'une importance fondamentale pour les rédacteurs de la constitution américaine bien qu'ils ne se faisaient pas d'illusion quant à la véritable nature de la constitution anglaise. Comme Madison le met en avant, « la constitution anglaise était à Montesquieu ce qu'Homer était aux écrivains didactiques de la poésie épique » (The Federalist Papers, no 47) », Alan Watson, Legal Transplants. An Approach to Comparative Law, Charlottesville, University Press of Virignia [31], p. 99.

${ }^{20}$ Soleil, S., « Le Modèle juridique français : recherches sur l'origine d'un discours », Droits [28], $\mathrm{n}^{\circ} 38$, p. 83 et s. ; Id., « Introduction », À la conquête du monde! La naissance du modèle juridique français, à paraître. V. aussi Boyer, P.-X., La réception..., op cit., p. 24.

21 Par actualisation, nous entendons deux choses : le fait de refaire correspondre le modèle (par définition décrivant un moment donné) à la réalité (qui elle est mouvante et dynamique). Sans cela, un modèle devient purement artificiel et conceptuel, dans le sens d'un modèle mathématique (V. sur ce sujet Badiou, A., Le concept de modèle, Paris, Maspero [2], p. 14 et s.) ; de plus, un modèle non déconnecté de la réalité, pour maintenir sa différenciation, doit sans cesse se modifier pour maintenir un caractère spécifique. Ainsi, le modèle français d'administration n'a eu de cesse de s'élaborer au contact d'autres modèles, notamment par l'étude de la doctrine allemande et britannique. V. sur ce sujet Sacco, R., $L a$ comparaison juridique..., op cit., pp. 113-128. V. aussi Meny, Y. (Dir.), Les politiques du mimétisme ..., op cit., 285 p. ; Boyer, P.-X., La réception..., op cit., not. pp. 42-43 et p. 63.

22 Par exemple, la Turquie a recopié le modèle français de laïcité. C'est d'ailleurs en ce sens que PierreXavier Boyer appréhende les modèles dans sa thèse La réception..., op cit. V. aussi, par ex.

Harith, A. D., «La réception du modèle juridique français par le Code civil irakien », Revue internationale de droit comparé, vol. 57, $\mathrm{n}^{\circ} 2$ [11], pp. 263-290. Le modèle, dans le sens du outil de reproduction, est doté d'une signification prescriptive, implémentée par le modélisateur, afin de procéder à des transferts juridiques ou à des convergences. 
prescriptive comme un étalon, que ce soit un but à atteindre ou un repoussoir. Il s'agit d'un argument d'autorité, ou du pathos. Enfin, le modèle est systématisation : il est question de reconstruire le monde rationnellement. Il y a donc une idéalisation en procédant à une mise en cohérence du système. Il doit être logique, et cela forme son $\operatorname{logos} .^{23}$ Ce faisant, il constitue un outil efficace-simplicité d'exposition, construction logique, vecteur de modification de l'existant—dans une analyse de droit comparé où la transplantation est désormais l'enjeu principal. ${ }^{24}$

9. Certes, cette critique est secondaire et tient davantage à un regret : l'intitulé de l'ouvrage amenait à croire que les modèles seraient l'objet crucial de l'étude. Cependant, Guillaume Tusseau ne semble tirer comme conclusions des raisons de l'échec des modèles de justice constitutionnelle que l'inadéquation de leur présentation actuelle. Il propose donc d'y substituer une typologie universelle. Au contraire, nous proposerons une remise en cause radicale des critères actuellement déployés.

10. Toute l'argumentation menée contre les modèles kelsénien et américain s'appuie sur la dénonciation du recoupement, par ces modèles, des critères typologiques. (pp. 26-50) Par exemple, pour révéler l'inanité de la dichotomie proposée, l'opposition classique du contrôle abstrait au contrôle concret est montrée comme non pertinente : «A plusieurs titres, il est possible de réfuter l'idée que les aspects concrets et abstraits du contrôle de constitutionnalité sont radicalement opposés. » (p. 37) En effet, « aux États-Unis, les tribunaux exercent-ils un contrôle abstrait de la constitutionnalité des lois et autres règles ? La réponse : un 'oui' massif. Étant donné la règle du 'case and controversy' établie par la Constitution américaine, à quel point le contrôle

\footnotetext{
23 Il est évidemment fait ici référence aux trois éléments constitutifs de toute rhétorique. Pour aller plus loin, Meyer, M., Principia Rhetorica, Paris, Fayard, coll. « Ouvertures » [17], 324 p.

${ }^{24}$ Le processus enclenché par la globalisation, la concurrence entre les droits sur fondée sur l'analyse économique du droit, la construction européenne, les démarches de la Commission et de la Cour de Justice de l'Union Européenne pour forger un droit commun, mais aussi des projets comme UNIDROIT tendent tous vers une interrogation autour de la transplantation juridique.

En parallèle, une grande réflexion vise, aujourd'hui, à remettre en cause la définition du droit comparé. Ainsi, Otto Pfersmann déplore que cette « discipline étrange [...] s'appuie toujours sur la plus faible des épistémologies » («Le droit comparé comme interprétation et comme théorie du droit », RIDC [21], n. 2, vol. 53, p. 275). Dans un sens identique, Esin Örücü consacre un ouvrage pour le cerner (The Enigma of Comparative Law, Leiden, Martinus Nijhoff Publishers [19], vi+242 p., not. Chap. 1, 8 et 9). Il ressort des débats deux positions typiques : celle considérant le droit comparé comme une discipline et donc un moyen de connaissance, au même titre que l'étude du droit civil ou commercial ; celle pour qui le droit comparé n'est qu'une méthode et non un objet, et donc qui doit être au service d'un objectif. Cet objectif oscille, selon ses formes, entre la recherche d'un droit commun et celle d'un regard critique des solutions juridictionnelles par l'analyse des réponses apportés à des questions apparemment similaires. Le point médian est la question du transfert juridique ou transplantation. C'est aujourd'hui autour de ce problème que se situent le débat en droit comparé, opposant ceux qui voient, dans le processus de réception et d'influence l'objet de la comparaison et ceux qui, par le recours aux traditions juridiques, contestent la possibilité même de ce processus. V. ainsi Sacco, R., La comparaison juridique..., op cit. pp. 113-128; Teubner, G., «Legal Irritants: Good Faith in British Law or How Unifying Law Ends Up in New Differences », Modern Law Review, vol. 61 [29], pp. 11-32; et surtout Watson, A., Legal Transplants..., op cit. et toute la discussion qui en suivit, not. entre Pierre Legrand, Lawrence Friedman, Roger Cotterrell et David Nelken dans la décennie -2010.
} 
américain est-il concret ? La réponse : bien peu. ${ }^{25}$ Dès lors, la conclusion est imparable : « Le critère [de la dimension abstraite ou concrète du contrôle] n'autorise dès lors rien de plus que la perception de nuances, sans permettre une véritable classification des institutions positives, et a fortiori l'élaboration d'un modèle. » (p. 40)

11. Aussi, la conséquence radicale serait d'abandonner ces critères et en proposer des nouveaux. Or, en fin d'ouvrage, dans l'annonce programmatique de la méthode employée, Guillaume Tusseau liste une série de critères à employer « à titre purement illustratif et non exhaustif, [tel] le fait [que le contrôle] vise la loi en tant que telle ou bien son application à un cas concret ». (p. 84) Certes, ce critère est plus précis qu'un simple contrôle abstrait ou concret, mais la finalité et la conséquence de ces deux critères demeurent identiques. Pour paraphraser Jean Rivero : que dirait-on du bûcheron qui abattrait l'arbre, mais qui se refuserait à couper les racines de l'arbre, laissant ce soin à la tempête d'hiver? ${ }^{26}$ Et pourtant, tout indique que chacun des critères peut fonder un nouveau modèle tout aussi néfaste que ceux combattus par Guillaume Tusseau. Ainsi, il consacre un paragraphe (\$2.2, section 1, chapitre III, pp. 75-77) spécifiquement à la dénonciation des modèles de Michel Fromont, ce dernier se fondant sur l'opposition abstrait-concret.

12. Et cette critique est généralisable à la quasi-totalité des critères cités par l'auteur dans son annonce programmatique. Certes, ils le sont à titre «purement illustratif »-il est possible de supposer qu'ils ne seront pas réellement employés_, mais aussi de manière «non exhausti[ve]». Cela laisse craindre que le projet ne soit pas aussi radical qu'espéré : en procédant à une typologie exhaustive, sont mélangés les éléments juridiques déterminants et ceux fongibles. ${ }^{27}$ En maintenant des critères qui sont erronés, comment peuton obtenir une évaluation convenable? Sans relancer un débat sur la pertinence de l'évaluation—elle l'est, dans l'absolu—, il est clair que la définition des critères prédétermine le résultat qui sera recueilli. De plus, à vouloir établir une typologie la plus neutre possible, il semble produire davantage une liste non ordonnée-une « checklist » dirait-on outre-Manche-à partir de laquelle le réel travail théorique débutera. À l'instar de l'historien qui met à jour et indexe une immense masse documentaire, son travail de thèse ne commence qu'à partir du moment où il met en relation les faits.

13. Enfin, il est étrange, pour un défenseur de la théorie réaliste de l'interprétation, de s'appuyer autant sur le texte constitutionnel et non sur sa pratique. ${ }^{28}$ Pour

\footnotetext{
25 Stone Sweet, A., Shapiro, M., « Abstract and Concrete Review in the United States », in Id., On law, Politics, and Judicialization, Oxford University Press, Oxford, 2002, p. 347, cité par Tusseau, G. p. 42.

${ }^{26}$ La formulation originale est « Que dirait-on du bûcheron qui couperait les racines de l'arbre, mais se refuserait à l'abattre, laissant ce soin à la tempête d'hiver ? » : Rivero, J., « Le Huron au Palais Royal ou réflexions naïves sur le recours pour excès de pouvoir », in Pages de doctrine, t. 2, p. 331.

27 V. Constantinesco, L.-J., Traité des droits comparés, t. III, Paris, Economica [6], p. 227 et s. Guillaume Tusseau a, par ailleurs, déjà éliminé plusieurs critères non pertinents pour une recherche juridique. (p. 84).

28 En effet, la plupart des exemples « exotiques » ne sont appuyés que par l'article de la Constitution prévoyant un tel dispositif.
} 
ses chefs de file, tels Michel Troper ${ }^{29}$ et Olivier Cayla, ${ }^{30}$ la Constitution est un texte dénué de toute force normative. Cette dernière n'apparaît que dans l'acte de volonté de l'interprète authentique. Par conséquent, il est dérisoire de rechercher dans le texte constitutionnel américain l'expression du contrôle de constitutionnalité. Cela rejoint à la fois la logique de Roscoe Pound qui opposait le « droit des livres » au « droit en action », mais aussi à la démarche bien acquise en droit comparé qu'est celle des formants juridiques. Rodolfo Sacco démontre ainsi qu'une analyse rapide se contentant d'un aspect du formant-le texte normatif, la jurisprudence ou la doctrine-ne suffit pas pour comprendre le mécanisme. Seule une vision d'ensemble permet de le saisir réellement et de ne pas procéder à des conclusions hâtives. ${ }^{31}$ Il est cependant aisément compréhensible qu'une comparaison en profondeur de chaque formant du panel mondial soit difficile à effectuer.

14. Le travail de Guillaume Tusseau appelle à une réflexion épistémologique sur les outils classiquement utilisés en droit comparé. Il sonne le glas des tentatives visant à maintenir artificiellement en vie un paradigme juridique mourant. Le modèle en tant que métaphore vive et le rejet des critères classiques de la typologie du droit constitutionnel comparé appellent l'avènement d'un nouveau paradigme, qualifié par Benoît Frydmann de post-moderne. ${ }^{32}$

Open Access This article is distributed under the terms of the Creative Commons Attribution Noncommercial License which permits any noncommercial use, distribution, and reproduction in any medium, provided the original author(s) and source are credited.

\section{Bibliographie}

1. Amalric, J.-L. 2006. Ricoeur, Derrida. L'enjeu de la métaphore. Paris: PUF, coll. « Philosophies ».

2. Badiou, A. 1969. Le concept de modèle. Paris: Maspero.

3. Bourdieu, P., J.-C. Chamboredon, and J.-C. et Passeron. 1968. le métier de sociologue. Paris: Mouton-Bordas.

4. Boyer, P.-X. 2002. La réception des modèles étrangers dans le droit constitutionnel français. Grande Bretagne et Etats-Unis au prisme de la réflexion française, Thèse, Rouen.

5. Cayla, O. 1999. « La chose et son contraire (et son contraire, etc.) ». Les études philosophiques, $\mathrm{n}^{\circ} 3$, pp. 291-310.

6. Constantinesco, L.-J. 1983. Traité des droits comparés, t. III. Paris: Economica.

7. Derrida, J. 1972. Marges de la philosophie. Paris: Éd. de Minuit.

\footnotetext{
${ }^{29}$ Troper, M., «Une théorie réaliste de l'interprétation », in Id., La théorie du droit, le droit, l'État, Paris, PUF, coll. « Léviathan » [30], p. 74.

${ }^{30}$ Cayla, O. « La chose et son contraire (et son contraire, etc.) », Les études philosophiques [5], n 3, p. 291 et s.

31 R. Sacco, La comparaison ..., op cit., p. 36. Il prend ici l'exemple de savoir si « l'acte à titre onéreux que l'héritier apparent conclut pour aliéner un bien successoral en faveur d'un tiers de bonne fois est valable » en France, en Belgique et en Italie. Dans les trois pays, la seule lecture du code offre une convergence différente de celle apportée par la pratique. De plus, l'occurrence d'un résultat identique ne permet même pas de conclure à l'identité des systèmes juridiques comparés.

32 Frydmann, B., « Y a-t-il en droit des révolutions scientifiques? », Journal des Tribunaux, n. 5827 [10], p. 813 .
} 
8. Derrida, J. 1987. Psychée. L'invention de l'autre. Paris: Galilée.

9. Eisenmann, C. 1986. La justice constitutionnelle et la Haute Cour constitutionnelle d'Autriche, av.prop. Vedel, G., préf. Kelsen, H., Annexe Favoreu, L., Paris, Economica, PUAM, coll. « Droit public positif », 1928, rééd.

10. Frydmann, B. 1996. «Y a-t-il en droit des révolutions scientifiques?». Journal des Tribunaux, n. 5827 , pp. 809-813.

11. Harith, A.D. 2005. « La réception du modèle juridique français par le Code civil irakien ». Revue internationale de droit comparé, vol. 57, n², pp. 263-290.

12. Heidegger, M. 1998. Logik als die Frage nach dem Wesen der Sprache. Francfort: Klostermann Vittorio GmbH, coll. « Gesamtausgabe II » $\mathrm{n}^{\circ} 38$.

13. Kelsen, H. 1942. "Judicial Review of Legislaton. A Comparative Study of the Austrian and the American Constitution ». The Journals of Politics, Vol. 4, pp. 183-200.

14. Meny, Y. (Dir.). 1993. Les politiques du mimétisme institutionnel. La greffe et le rejet. Paris: L'Harmattan, coll. « Logiques politiques ».

15. Meyer, M. 2008. De la problématologie. Paris: PUF, Coll. « Quadrige ».

16. Meyer, M. 1999. Pour une histoire de l'ontologie. Paris: PUF, coll. « Quadrige».

17. Meyer, M. 2008. Principia Rhetorica. Paris: Fayard, coll. « Ouvertures ».

18. Nietzsche, F. 1969. Livre du philosophe, trad. Kremer-Marietti, A., Paris, Aubier-Flammarion.

19. Örücü, E. 2004. The enigma of comparative law. Leiden: Martinus Nijhoff Publishers.

20. Ost, F. 2007. Dire le droit, faire justice. Bruxelles: Bruylant, coll. « Penser le droit».

21. Pfersmann, O. 2001. «Le droit comparé comme interprétation et comme théorie du droit ». RIDC, n. 2, vol. 53, pp. 275-292.

22. Quine, W.V.O. 1961. From a logical point of view, 2e éd. New York: Harper Torchbooks, 1953.

23. Ricoeur, P. 1975. La métaphore vive. Paris: le Seuil, Coll. « L'ordre philosophique ».

24. Rivero, J. 1951. « Apologie pour les faiseurs de systèmes ». D., Chronique, pp. 99-102.

25. Rivero, J. « Le Huron au Palais Royal ou réflexions naïves sur le recours pour excès de pouvoir », in Pages de doctrine, t. 2, pp. 329-334.

26. Sacco, R. 1991. La comparaison juridique au service de la connaissance du droit. Paris: Economica, coll. «Études juridiques comparatives ».

27. Soleil, S. «Introduction », À la conquête du monde ! La naissance du modèle juridique français. à paraître.

28. Soleil, S. 2003. « Le Modèle juridique français: recherches sur l'origine d'un discours ». Droits, $\mathrm{n}^{\circ} 38$, pp. 83-96.

29. Teubner, G. 1998. « Legal Irritants: Good Faith in British Law or How Unifying Law Ends Up in New Differences ». Modern Law Review, vol. 61, pp. 11-32.

30. Troper, M. 2001. La théorie du droit, le droit, l'État. Paris: PUF, coll. « Léviathan ».

31. Watson, A. 1974. Legal transplants. An approach to comparative law. Charlottesville: University Press of Virignia.

32. Weber, M. 1965. Essais sur la théorie de la science, trad. Freund, J., Paris, Plon, Coll. « Recherches en sciences humaines $\gg \mathrm{n}^{\circ} 19$.

33. Zoller, E. 2000. «Qu'est-ce que faire du droit constitutionnel comparé?». Droits, $\mathrm{n}^{\circ} 32$, pp. 121-134. 